\title{
5 Fazit: Von Kontinuitäten und Wandlungen in der japanischen Literaturtheorie von „Fiktion“ und „Wirklichkeit“
}

In Abschnitt 3.3.1 wurden die von japanischen Literaturtheorien bis 1850 realisierten inhaltlichen Schwerpunkte des Fiktiven (im Iserschen Sinne) zunächst in einer Übersicht zusammengefasst. Das (nicht funktional synchron gedachte) „System“ war entelechisch zu verstehen. Am Schluss der vorliegenden Abhandlung steht nun die Beantwortung der mit diesem System zusammenhängenden, folgenden Fragen an.

- Wurden nach 1850 eventuelle Inkonsistenzen dieses Systems beseitigt und offene Stellen geschlossen?

- Wurde das im Jahr 1850 sichtbare Potenzial zur Weiterentwicklung eines Systems genutzt?

- Wurde ein alle Aspekte der Produktion und Rezeption fiktionaler Literatur erfassendes, integrales literaturtheorisches System geschaffen?

- Welche Rolle nimmt das Fiktive in japanischen Literaturtheorien zum Ende des Betrachtungszeitraums ein?

\subsection{Veränderungen in der literarisch referenzierbaren extratextuellen Welt}

Im Betrachtungszeitraum verändern sich in der Folge der historischen Entwicklungen die Konkreta in der referenzierbaren Gegenwart teilweise drastisch: Dampfschiffe, Flugzeuge, Straßenfahrzeuge und andere technische Geräte kommen zu den bisher genutzten dazu oder ersetzen sie gar. Die Frage, ob auch die vielfach referenzierten Konkreta der Natur (etwa Flora, Fauna und der Mensch) sich im Betrachtungszeitraum physisch grundlegend wandeln, sprengt den Rahmen der vorliegenden Abhandlung. Es ist selbstverständlich, dass auch die Abstrakta als Teil der referenzierbaren extratextuellen Welt sich massiv verändern, solange sie (wie im erwähnten entelechischen System) als Aspekt des Physischen begriffen werden. Das betrifft die Religion ebenso wie Philosophie und Naturwissenschaft der extratextuellen Welt, und natürlich liegt es auf der Hand, dass diese Veränderungen sich auch auf die Protagonisten der Literaturgeschichte in ihrer praktischen Produktion und deren theoretischer Begleitung auswirkten. Es ist hier nicht der Ort, die Einflüsse ausländischer Literaturen im Detail zu besprechen. Es mag auch ausreichen, auf den Einfluss der in japanischen Übersetzungen ab der 
Meiji-Restauration erstmals einem breitem Publikum zugänglich gewordenen literarischen Produktion des Westens hinzuweisen. ${ }^{561}$ Dass diese Übersetzungsliteratur als Teil der massiven Veränderungen der extratextuellen, in der Literatur referenzierbaren Realität wahrgenommen wurde, ist einhellige Meinung der Literaturhistoriker.

\subsection{Das literaturtheoretische System „Literatur“ am Ende des Betrachtungszeitraums}

Das System „Literatur“ subsumierte zu Beginn des Betrachtungszeitraums unter dem Wort bungaku 文学 („Schriftgelehrsamkeit“) verschiedene Teilbereiche der Wissenschaften, unter denen der neokonfuzianischen Philosophie und dem Buddhismus Schlüsselrollen als vom Staat sanktionierte Erklärungen der Welt zukamen. Beide Erklärungsmodelle waren handlungsorientiert und darum zur Schaffung ethischer und moralischer Kodices geeignet, welche in die Vielfalt menschlicher Aktivitäten auch die des Schreibens und Lesens einschlossen.

Die neokonfuzianisch orientierte Literaturtheorie propagierte zu Anfang des Betrachtungszeitraums - bei allen Varianten in Detailfragen - die monistische Vorstellung von einem die gesamte Welt umfassenden Naturprinzip ri 理 (chin. li), das die Ausprägungen des spezifischen Äthers $k i$ 気 (chin. qi) steuert und auf allen denkbaren Ebenen die Entwicklungen beeinflusst: die Konstellation der Planeten und den Zyklus der Jahreszeiten ebenso wie die zwischenmenschlichen Beziehungen. „Literatur“ zu verfassen bedeutete in diesem Rahmen zu Anfang des Betrachtungszeitraums, die objektive Wahrheit des Zusammenhangs zwischen vorhandenen, spezifischen Phänomenen der Erscheinungswelt und dem Naturprinzip ri aufzudecken und Handlungsanweisungen daraus $\mathrm{zu}$ entwickeln. Es nimmt nicht wunder, dass die Herstellung (lat. fictio) bis dahin nicht vorhandener Phänomene nicht Aufgabe der „Literatur“ im klassisch-neokonfuzianischen Sinne sein konnte.

Die buddhistisch orientierte Literaturtheorie vertrat zu Anfang des Betrachtungszeitraums die Vorstellung, die (bei neokonfuzianisch orientierten Literaturtheorien im Mittelpunkt stehende!) Erscheinungswelt sei transzendent und für sich genommen volatil, die Erkenntnis einer dahinterliegenden Wahrheit nicht möglich, weil zwischen betrachtendem Subjekt und betrachtetem Objekt keine Trennung bestehe. Eine wichtige Aufgabe des Schrifttums sah zu Beginn des Betrachtungszeitraums die buddhistisch orientierte Literaturtheorie folglich 
darin, die Einheit von Subjekt und Objekt der Wahrnehmung in der Literatur klarzulegen.

Zum Ende des Betrachtungszeitraums gerät das bis dahin feste Gefüge der Geisteswelt auch in den Literaturtheorien aus den Fugen. Die neokonfuzianisch orientierte Textgelehrsamkeit wird nun dafür kritisiert, dass sie angeblich die Handlungsorientierung verloren habe und zu einer Kommentarwissenschaft verkommen sei, welche mit der unter dem Einfluss des westlichen Auslands komplexer gewordenen Realität nicht mehr zurechtkomme. Unter „Wissenschaft“ wird nun vielfach einengend die Naturwissenschaft verstanden, die in ihrer positivistisch verstandenen Weltsicht klare Orientierungen und Handlungsanweisungen zu versprechen scheint. „Literatur“ emanzipiert sich gleichzeitig als Teilgebiet eines autonomen Feldes „Kunst“, welches sich zunehmend von der Handlungsorientierung des gesellschaftlichen Alltags entfernt.

Was zu Beginn des Betrachtungszeitraums allein die Aufgabe der „Wissenschaft“ (gakumon 学問 im Sinne einer der Geisteswissenschaft nahestehenden Textgelehrsamkeit) war, wird zum Ende zumindest in der Literaturtheorie auch eine Aufgabe der fiktionalen Literatur: die Aufdeckung dessen, was die physische und die metaphysische Welt im Innersten zusammenhält. Allerdings wird die Aufgabe dabei spezifiziert: Von Theoretikern wird Literatur nun als ein Mittel zur Erkenntnis derjenigen Zusammenhänge beschrieben, welche der zunehmend an der Naturwissenschaft orientierten, positivistischen modernen Wissenschaft verborgen bleiben müssen. Literatur beschränkt sich in der sie begleitenden Theorie nicht mehr darauf, behauptete und verifizierte Fakten der Erscheinungswelt zu beobachten und zu analysieren, sie leistet vielmehr die Herstellung (lat. fictio) neuer Wirklichkeiten jenseits der Welt klassischer Fakten und Erscheinungen. Fiktionale Literatur überschreitet in der Theorie auf diese Weise jenen traditionell festgeschriebenen Akzeptanzrahmen, der ihr meist nur moralische Unterweisung und Unterhaltung zugesteht, und wird $\mathrm{zu}$ einer erkenntnisreichen Überschreitung überkommener Wahrheitsbegriffe.

\subsection{Altes und Neues in der Literaturtheorie am Ende des Betrachtungszeitraums}

\subsubsection{Literaturtheorie diversifiziert sich am Ende auf evolutionärem Weg}

So wenig sich die Innen- und Außenpolitik oder soziale und kulturelle Institutionen Japans durch das Auftauchen der „Schwarzen Schiffe“ im Jahr 1853 als bedrohliche Präsenz ausländischer Mächte mit einem Schlage vollkommen verändert haben, so wenig taten sie es am 7. Januar 1868 durch die endgültige 
Zurückdrängung der Feudalmächte und durch die Verkündung der Restauration der Kaisermacht, und so wenig taten sie es auch mit der ersten Sitzung des japanischen Parlaments am 25. November 1890. Und ebenso wenig änderten sich Praxis und Theorie der Literatur von einem auf den anderen Tag grundlegend. Wir haben es vielmehr im Betrachtungszeitraum auch auf dem Gebiet der Theorie der Literatur mit einer evolutionären Diversifizierung und zunehmend komplexen Überlagerung von Strata zu tun. ${ }^{562}$

Was nun am Ende des Betrachtungszeitraums zu dem von Natsume Sōseki in Bungaku ron (1907; Abschn. 4.4.3) entworfenen System aus literaturtheoretischer Sicht noch fehlt, lässt sich wie folgt zusammenfassen.

\subsubsection{Auch 1890 noch ein Desideratum: Rezeption als neuer Aspekt der Literaturtheorie}

Das Lesen und der Leser als Individuum sind eine Sache - eine andere die abstrakte Rolle des Rezipienten in der prozessual gedachten Theorie von der Literatur. Lesen und Leserschaft als Individuen werden im Verlauf der Kommerzialislierung der fiktionalen Literatur Japans immer intensiver als Kunden oder Zensoren angesprochen. ${ }^{563}$ Für den Betrachtungszeitraum sei als Beispiel Kanagaki Robun (Abschn. 4.1.5) genannt. Robun sieht sich zurecht der Tradition der Edo-Zeit verhaftet und darum zu Beginn der Meiji-Zeit angesichts der massiven gesellschaftlichen Veränderungen Japans dem Druck ausgesetzt, seine traditionelle fiktionale Produktion zu rechtfertigen. Im Bewusstsein, dass die traditionelle gesaku-Literatur nur überleben kann, wenn sie sich im Hinblick auf aktuelle gesellschaftliche Aufgaben als nützlich erweist, appelliert er an die Zensur und die Mächtigen, die Nützlichkeit der gesaku zur Kenntnis zu nehmen. Das verlangt ein Umdenken der Leser, nicht der Autoren, und doch sind damit kaum Autor und Leser in ihren gesellschaftlichen Bezügen oder ihrer Rollendisposition und den daraus folgenden Spielarten der Rezeption, geschweige denn des Fiktionalen angesprochen. Eine Differenzierung der theoretischen Begleitung der Literatur war mit solchen Überlegungen nicht verbunden - es kam Robun und anderen auf die Akzeptanz ihrer Texte an, nicht auf deren Bezug zu dem, was die Welt im Innersten zusammenhält.

562 Es sei in diesem Zusammenhang noch einmal auf MAEDA 1972 (Anm. 24) verwiesen.

563 Ekkehard May bietet eine umfangreiche und durch zahlreiche Textzitate veranschaulichte Darstellung der Mittel zur Ansprache der Leser und zur Bindung des Leserpotenzials (MAY 1983: 150-199). 
Prinzipiell ändert sich innerhalb des Betrachtungszeitraums daran nichts. Dies mag zunächst überraschen, denn das schon Ende des 17. Jahrhunderts im Umfeld von Matsuo Bashō thematisierte Verhältnis des betrachtenden Subjekts zum betrachteten Objekt rückt neben dem Prozess der Produktion ja auch den des Abstraktums „Rezipient“ ins Blickfeld. Ein Ausbau des schon von Bashō angelegten literaturtheoretischen Ansatzes wäre möglich gewesen. Doch stattdessen sind die Literaturtheoretiker zum Ende des Betrachtungszeitraums vorrangig mit der Neuformulierung des Systems „Literatur“ in seiner Gesamtheit und seiner Gegenstände befasst. Maeda Ai sieht erste Ansätze zu einer japanischen Theorie des Lesers (dokusha ron 読者論) erst gegen Mitte der 1920er Jahre. Diese Form der Literaturtheorie habe die bis dahin vorherrschende impressionistische Literaturkritik, wie sie sich etwa in den Gesprächen literaturkritischer Zirkel in der Zeitschrift Shinchō manifestierte, abgelöst. Als prominente Vertreter der modernen, außerhalb der literarischen Produktion oder der Literaturkritik stehenden Literaturtheorie sieht Maeda die Marxisten Aono Suekichi 青野季吉 (1890-1961) und Katagami Noburu片上伸 (1884-1928). Als erste leserzentrierte Literaturtheorie habe, so Maeda, Katagamis in der Zeitschrift Kaizō 改造 veröffentlichter Aufsatz Bungaku no dokusha no mondai 文学の読者の問題 ([Das Problem des Lesers der Literatur], April 1926) zu gelten. ${ }^{564}$

\subsubsection{Autoreferentialität auch 1890 noch ohne literaturtheoretische Basis}

Was nun aber am Ende des Betrachtungszeitraums entstandene Literaturtheorien von ihren Vorgängern nicht unterscheidet, ist das darin zum Ausdruck kommende Verhältnis $\mathrm{zu}$ jener von Iser als Wirkungsbedingung literarischer Texte beschriebenen Unbestimmtheit. ${ }^{565}$ Sie wird von den Theoretikern noch 1890 offenbar nur akzeptiert, wenn das aus ihr erwachsende Fiktionale ein extratextuelles Referens hat. Die Schaffung einer neuen „Welt“ ohne Bezug zu einer bereits vorhandenen ist in dieser Zeit noch nicht Gegenstand einer Literaturtheorie.

Zum Ende des Betrachtungszeitraums stellt sich Literatur in der Theorie noch immer als Begleitung der extratextuellen, „realen“ Welt oder als Verweis auf sie dar. Zwar werden der metaphysische und der physische Teil der Realität in der Literaturtheorie deutlicher gegeneinander abgesetzt, die Theorie macht aber die Literatur selbst nicht zu einem Teil referenzierbarer Realität. Diese Au-

564 MAEDA 1973: 313; KatAGAMI 1997 [1926].

565 Siehe S. 6-7. 
toreferenzialität des literarischen Textes wird erst später zu einem Gegenstand der japanischen Literaturtheorie.

\subsubsection{Das Phänomen des Rekurses auf ältere literaturtheoretische Modelle}

$\mathrm{Zu}$ den komplexesten Phänomenen der japanischen Literaturtheorie im Betrachtungszeitraum gehört der in seiner Zitathaftigkeit deutlich als solcher gekennzeichnete Rekurs auf ältere literaturtheoretische Modelle. Komplex sind solche Theorien, weil sie Funktionen haben, die über den unmittelbar argumentativen Zweck hinausgehen.

Dass die Verwendung von Wörtern wie kokoro („Herz“), die in der japanischen Literaturtheorie vielfach und mit sehr verschiedenen Begriffsinhalten eingesetzt werden, nicht in jedem Falle als Zitat verstanden werden können, liegt auf der Hand: Denn die kleinste überhaupt als Zitat erkennbare Einheit ist das Theorem als Teil einer Theorie, also etwa die argumentierende Zuschreibung von Begriffsinhalten des Wortes kokoro. Von solchen das Wort kokoro beinhaltenden Theoremzitaten sind die nach dem Kokinshū (914) entstandenen Literaturtheorien gleichsam übersät, gleich ob es sich um echte oder chiffrierte Zitate handelt. ${ }^{566}$

Nun könnten Theoremzitate stets als sentimentale, eskapistische Rückwendung verstanden werden, und in der Tat finden sich in den hier zitierten Theorien häufig Klagen über vermeintliche Verfallserscheinungen der Gegenwart, denen der Theoretiker ästhetische Systeme der Vergangenheit entgegensetzt. Ungleich ergiebiger ist es jedoch, solche Zitate als Verweis auf die grundsätzliche Bedeutung literaturtheoretischer Begrifflichkeiten, der aus ihnen entwickelten Argumentationen sowie und vor allem auf den Sinn des literaturbezogenen Theoretisierens $\mathrm{zu}$ verstehen. Wir kommen damit beinahe von selbst $\mathrm{zu}$ den im Zusammenhang mit Tsurayuki (dem Verfasser des literaturtheoretischen Vorworts des Kokinshū) und Hegel (S. 97) bereits angeklungenen Fragen zurück, nämlich: Was können, wollen und sollen wir äußern, wie können, wollen und sollen wir es äußern, und warum äußern wir uns überhaupt? Genau diese Fragen bilden, gewissermaßen als kulturanthropologische Konstante, den Hintergrund des im folgenden Abschnitt hervorgehobenen Rekurses auf eine damals beinahe zweihundert Jahre alte Quelle.

566 Echte Zitate sind durch explizite Signale der Zitathaftigkeit (im vormodernen Japanischen etwa durch Formulierungen wie to iikeri im Sinne von „so sagte er“), chiffrierte Zitate durch Angaben zu Autor und Titel, sowie durch den Einsatz stilistisch fremder Sprachen (etwa kanbun) und ästhetischer Verfahren gekennzeichnet (Grundsatzfragen der Zitathaftigkeit diskutiert ORAIĆ TOLIĆ 1995). 


\subsection{Die Fiktion als Mimesis der Idee}

Ein besonderes Beispiel für den Rekurs auf ältere literaturtheoretische Modelle haben wir im Jōkyōshiki kaiin roku 貞享式海印録 ([Verzeichnis von Abdrucken auf dem Meere im Stile der Jōkyō-Zeit], 1859) des Harada Kyokusai (Abschn. 4.3.2) kennengelernt. Harada verweist darin auf die Einheit von betrachtendem Subjekt und betrachtetem Objekt sowie auf die „Idee“ (im Sinne einer versprachlichten Vorstellung von außersprachlichen Objekten) als die eigentliche Wirklichkeit.

Nun ist es wohl faszinierend, dass sich in diesem konkreten Fall eine Art von Idee-Begriff von der 1694 entwickelten Theorie des Haikai-Dichters Matsuo Bashō über die von seinem Schüler Kagami Shikō unternommene Verdichtung (gedruckt postum 1736) und von dort aus über Kyokusai (1859) bis hin zu Futabatei Shimei (1886), Mori Ōgai und Ishibashi Ningetsu (beide 1890) wie ein roter Faden durchzuziehen scheint. Indes: Faszinierender noch ist die Frage, welche in japanischen Literaturtheorien für das Fiktionale ausgemachten Inhalte über das „Nichtseiende“ als Begriff für die wirkmächtige Idee hinausgehend unabhängig von historischen Kontexten als Gegenstand literarischer Mimesis unentbehrlich schienen und scheinen. Und damit sind wir zur eingangs der Abhandlung angeklungenen Vorstellung von einer „Tauglichkeit literaturtheoretischer Begriffe zur Beschreibung ideengeschichtlicher Prozesse“ zurückgekehrt, ${ }^{567}$ in deren Rahmen Mimesis sich als kulturanthropologische Konstante darstellt. ${ }^{568}$

Insofern der Dichotomie „Leere - Fülle“ ursprünglich ein monistischer Begriff von „Wahrheit“ zugrundelag, versteht sich von selbst, dass im Lichte der in den vorausgehenden Abschnitten angedeuteten literaturhistorischen Entwicklung die Literaturtheorien gegen Ende des Betrachtungszeitraums die Vorstellung von einer „objektiven Wahrheit“ durch die Vorstellung einer „Idee“ ersetzen und überdies dynamisieren: Literatur deckt in den entsprechenden Theorien nicht mehr eine für alle Zeiten und für jedes Phänomen gültige Wahrheit auf, sondern wird Mimesis des Kernmaterials unendlich vieler dialektischer Prozesse, die eine wechselseitige Beeinflussung von Kunst und Gesellschaft prägen.

Anders gesagt: Die Begriffsinhalte der Dichotomie „Leere - Fülle“ waren zu Anfang des Betrachtungszeitraums festgeschrieben in der Beziehung von „Leere“ und „Fülle“ noch fest in ihren Werten. „Leere“ beinhaltete etwa „Lüge“ oder „Wahrheit“ (!), „Metaphysisches“ oder „Formloses“, demgegenüber beinhaltete „Fülle“ etwa „Wahrheit“, „Physisches“ oder „Formhaftes“. Gegen Ende des Betrachtungszeitraums zeichnet sich eine Dynamisierung der Begriffsinhalte insofern

567 ZEUCH 2010. Siehe S. 3.

568 ZEUCH 2010. 
$\mathrm{ab}$, als sie teilweise oder gar nicht festgelegt werden. Die Literaturtheorie spricht der Literatur nun die Ingangsetzung eines hermeneutischen Zirkels zu: Eine der beiden Stellen (ob nun „Fülle“ oder „Leere“, ob „Fiktion“ oder „Wirklichkeit“) ist demnach besetzt und dient als Ausgangspunkt der Erforschung der jeweils anderen, unbesetzten Stelle. Literatur wird auf diese Weise zu einem Instrument der Investigation noch unbekannter oder Kreation noch nicht existierender Welten. In der literarischen Praxis dürften die Synästhesien in der Haikai-Poesie des Matsuo Bashō als frühe Beispiele der Unbesetztheit einer der beiden Stellen („Fülle“ oder „Leere“) gesehen werden. Erste Ansätze zu einer theoretischen Definition der Literatur als investigatives und kreatives Instrument zeigt sich ab etwa 1870 in Texten wie Nishi Amanes Hyakugaku renkan (1870-72; siehe Abschn. 4.1.6). Einen vorläufigen Höhepunkt im Niveau der literaturtheoretischen Formulierung setzte im Betrachtungszeitraum Mori Ōgai mit Texten wie ,Bungaku to shizen“ wo yomu (1890; Abschnitt 4.4.2).

Zur durch den literarischen Text ohne „Weltbezug“ neu geschaffenen Realität gehört dann die Auflösung referentialisierbarer Entitäten wie etwa in der Miszellensammlung Aru ahō no isshō 或阿呆の一生 (Das Leben eines Narren, 1927) von Akutagawa Ryūnosuke 芥川龍之介 (1892-1927). Im Folgenden ein Beispiel daraus.

28 - Mord Die Provinzstraße lag im Licht der Sonne, über ihr schwebte der Gestank von Kuhmist. Er wischte sich den Schweiß ab und stieg die Straße weiter bergan. An beiden Seiten der Straße verströmte reifer Weizen einen kräftigen Duft. „Töte! Töte!“ Wieder und wieder erklang dieses Wort in seinem Kopf. Wen wollte er töten? - Für ihn lag es auf der Hand. Er erinnerte sich an den unterwürfigen und schmierigen Mann mit den kurzgeschnittenen Haaren. Über dem strohgelben Weizen tauchte ein katholisches Kirchenschiff auf, dann die gewölbte Kuppel. ${ }^{569}$

Diese in sich abgeschlossene Miniatur lässt keinerlei Ko- oder Kontext zu den anderen Teilen der Miszellensammlung erkennen. Akutagawas Text hält damit genau jene Unbestimmtheiten bereit, die Wolfgang Iser wegen ihrer Appellstruktur als wichtigste Wirkungsbedingung literarischer Texte sieht - Unbestimmtheiten von bis dahin noch nicht einmal in der Lyrik von Matsuo Bashō gekannten Dimensionen.

569 AKUTAGAWA-PUTZ 1997 [1927]: 39. Original: 二十八 殺人 /田舎道は日の光の中に牛の粪の 臭気を漂はせてるた。彼は汗を拭ひながら、爪先上がりの道を上って行った。道の両側に熟 した麦は香ばしい匂を放ってるた。「殺せ、殺せ。……….彼はいつか口の中にかう云ふ 言葉を繰り返してるた。誰を?--—-ーそれは彼には明らかだった。彼は如何にも卑屈らし い五分刚りの男を思ひ出してるた。すると黄ばんだ麦の向こうに羅馬カトリック教の伽藍が 一宇、いつの間にか円屋根を現し出した。 .......... (NKiBT 38: 254). 
Doch wie sieht die Entwicklung in der Theorie der Literatur aus? Zu Anfang des Betrachtungszeitraums wollen, können oder sollen die Literaten aus der Sicht der Literaturtheoretiker als objektiv betrachtete Wahrheiten äußern, die Autoren können, wollen oder sollen sie in einer Verbindung von „Fülle“ und „Leere“ äußern, und sie tun dies alles überhaupt, um selbst eine Position zu dieser Wahrheit einzunehmen. Zum Ende des Betrachtungszeitraums rückt der Leser in seiner Rolle als Interpret offener Beziehungen zwischen „Fülle“ und „Leere“ in das Blickfeld - die explizite literaturtheoretische Formulierung des Phänomens der intratextuellen Kommunikation steht unmittelbar bevor. Vor diesem Hintergrund erhält die literarische Mimesis einen grundsätzlich anderen Stellenwert: Sie strebt zum Ende des beobachteten Prozesses hin zur Nachahmung desjenigen Vorgestellten, das nach Aristoteles „gemäß innerer Wahrscheinlichkeit oder Notwendigkeit geschehen würde, was als eine Handlung eines bestimmten Charakters möglich ist“. ${ }^{570}$ Um es anhand der erwähnten Begrifflichkeiten von Georg Lukács zu sagen: Die japanische Literaturtheorie schreitet im Betrachtungszeitraum von einer „desanthropomorphisierenden“ Zuschreibung von Wahrheitsbegriffen auf eine „anthropomorphisierende“ Weltsicht ${ }^{571} \mathrm{zu}$ - auch wenn sie diese Weltsicht erst in den folgenden Jahrzehnten erreicht.

570 „Aufgrund des Gesagten ist auch klar, dass nicht dies, die geschichtliche Wirklichkeit < einfach > wiederzugeben, die Aufgabe eines Dichters ist, sondern etwas so < darzustellen >, wie es gemäß innerer Wahrscheinlichkeit oder Notwendigkeit geschehen würde, d. h. was $<$ als eine Handlung eines bestimmten Charakters > möglich ist. Denn ein Historiker und ein Dichter unterscheiden sich nicht darin, dass sie mit oder ohne Versmaß schreiben (man könnte die Bücher Herodots in Verse bringen, und sie blieben um nichts weniger eine Form der Geschichtsschreibung, in Versen wie ohne Verse), der Unterschied liegt vielmehr darin, dass der eine darstellt, was geschehen ist, der andere dagegen, was geschehen müsste. Deshalb ist die Dichtung auch philosophischer und bedeutender als die Geschichtsschreibung. Die Dichtung nämlich stellt eher etwas Allgemeines, die Geschichtsschreibung Einzelnes dar.“ [1451a35-1451b5] (ARISTOTELES-SCHMitT 2010 [3. Jh. v. Chr.]: 13-14; die < >-Klammern umschließen Ergänzungen von Arbogast Schmitt).

571 S. 57. 
